\title{
Visual acceleration detection: Effect of sign and motion orientation
}

\author{
JACK B. CALDERONE \\ San Jose State University, San Jose, California \\ and \\ MARY K. KAISER
NASA Ames Research Center, Moffett Field, California
}

\begin{abstract}
Thresholds for the detection of constant acceleration and deceleration of a discrete object mov. ing along horizontal and vertical axes were studied. A staircase methodology was used to determine thresholds for three average velocities $(0.7,1.2$, and $1.7 \mathrm{deg} / \mathrm{sec})$. Thresholds, expressed as the proportion of velocity change, did not differ significantly among the average velocities; thus, a consistent Weber-like fraction is suggested by the data. Furthermore, there was an interaction between the axis of motion (horizontal or vertical) and the sign of the velocity change (acceleration or deceleration): accelerations were easier to detect along the vertical axis, decelerations along the horizontal axis.
\end{abstract}

Object motion is one of the principle topics of perceptual psychology, yet relatively little research has focused on the perception of nonuniform motion. Thus, for example, little is known about observers' ability to detect a linear change in an object's velocity (i.e., a constant acceleration). Early studies of acceleration perception (Geiger, 1903; Wundt, 1874) are difficult to interpret, due to methodological problems. Later studies, which are methodologically sound, provide valuable information about acceleration, yet they often confound acceleration perception with manual tracking (e.g., Gottsdanker, 1955), trajectory extrapolation (Jagacinski, Johnson, \& Miller, 1983; Rosenbaum, 1975), or other extraneous tasks.

The perception of acceleration has been characterized both as a direct (Rosenbaum, 1975) and as an indirect process (Filion, 1964; Gottsdanker, Frick, \& Lockard, 1961). Most models of indirect perception propose that observers compare the velocities of the initial and final motion segment (Regan, Kaufman, \& Lincoln, 1986). These models, however, need to reconcile observers' ability to detect accelerations with their ability to detect velocity differences. When presented with successive presentations of constant velocity stimuli, observers can detect about a 6\% difference (McKee, 1981; Orban, Wolf, \& Maes, 1984). Previous research (Schmerler, 1976) on

This article is based on Jack B. Calderone's honors' thesis. Portions of this paper were presented at the Fourth International Conference on Event Perception and Action, Trieste, Italy, August 1987, and at the 68th Convention of the Western Psychological Association, Burlingame, CA, April 1988. We thank Kenneth Barry of Sterling Software for his programming support and Walter Johnson for his helpful comments on an earlier version of this manuscript. Requests for reprints should be sent to Mary K. Kaiser, NASA Ames Research Center, Mail Stop 2393, Moffett Field, CA 94035. acceleration thresholds suggests a much greater value (79\%-105\%).

The present study examines observers' detection thresholds for linear acceleration and deceleration for motions along the principle visual axes. Some researchers have reported directional anisotropies for simple motion detection (Halloran, Clark, \& Stewart, 1976; McColgin, 1960 ), but the bias has not been consistent and may be due to positional as well as directional factors. In addition, sign anisotropies have been found for acceleration detection. Several researchers have reported that decelerations are easier to perceive than accelerations, but only horizontal motions were used in these studies (Gottsdanker et al., 1961; Schmerler, 1976). In the present study, both directional factors and accelerational sign are taken into account.

One difficulty in presenting the motion of discrete stimuli is that the manner in which motion is initiated can lead to nonveridical perceptions of velocity (Jagacinski et al., 1983; Runeson, 1974). Some researchers have attempted to circumvent these problems by using drifting sine-wave gratings as stimuli (Regan et al., 1986). However, this may alter the phenomenon of study from acceleration of a discrete object to changes in field flow rates. The stimuli in the current study emerged from behind a mask, traversed the screen without reversing direction, then were occluded by the mask on the opposite side. This avoids most of the motion characteristics that bias observers (for example, motion onset or termination), while it maintains a discrete object motion.

A final concern plaguing studies of acceleration perception has been the proper characterization of the acceleration parameter. Physics, of course, defines acceleration as change in velocity per unit time. This, however, seems an inappropriate psychophysical measure, since an 
observer's sensitivity is likely also to depend on the duration of the acceleration and the initial velocity of the object. Thus, researchers have found it more useful to characterize acceleration in terms of the overall change in velocity, either as a ratio of final to initial velocity (Schmerler, 1976) or as the proportion of change in velocity (Regan et al., 1986), thus:

$$
\left[V_{\text {final }}-V_{\text {inital }}\right] / V_{\text {average }} \text {. }
$$

This quantity is analogous to a Weber fraction, and it is the threshold measurement employed in the current study. Three average velocities were used in order to assess, within a limited velocity range, whether this fraction remains constant for acceleration-deceleration detection thresholds.

\section{METHOD}

\section{Observers}

Nine observers ( 5 males and 4 females) participated in the study. Their ages ranged from 19 to 25 years, and all had normal or corrected-to-normal binocular vision. The observers were paid for their participation.

\begin{abstract}
Apparatus
The stimuli were displayed on a 13 -in. monitor with a $60-\mathrm{Hz}$ noninterlaced, raster refresh rate driven by a Commodore Amiga 1000 microcomputer. A 5-mm white square served as the target object. The accelerations, which occurred throughout the entire traversal, were shown by varying the number of pixels moved between refreshes. The high refresh rate ensured that the motion of the target appeared continuous. A mask placed in front of the monitor screen created a $17.5-\mathrm{cm}$ square window. Both the mask and the monitor background were blue. The observers' judgments were recorded with a two-button mouse (a hand-held input device). The experiment was conducted in a sound-attenuated booth with minimal ambient lighting.
\end{abstract}

\section{Design}

A staircase methodology with decreasing step size was used to locate the observers' $X_{50}$ (point of $50 \%$ detectability) for each condition. ${ }^{I}$ Two blocks of staircases were run: one for the acceleration conditions, the other for deceleration. Each block consisted of three sets, one for each of three average velocities $(0.7,1.2$, and $1.7 \mathrm{deg} / \mathrm{sec}$ ). Each set contained eight interleaved staircases, crossing four directions of motion (up, down, left, right) with two initial acceleration values ( 0 or clearly suprathreshold). Thus, there were four within-observer factors: sign of acceleration, average velocity, motion direction, and point of staircase initiation. The order of blocks, sets, and staircases within sets was randomly determined for each observer. In addition, monitor orientation (upright or on its side) was counterbalanced across observers to disambiguate motion direction effects and screen artifacts. ${ }^{2}$

\section{Procedure}

The observers were seated approximately $170 \mathrm{~cm}$ from the monitor screen. Thus, the display subtended $6.0^{\circ}$ of the visual field, and the target square subtended approximately $0.2^{\circ}$. Head and eye position were unconstrained. On each trial, the stimulus spot appeared on the midline of the axis perpendicular to the direction of motion and translated the screen, initializing and completing its motion outside the observers' field of view. The observers were instructed to judge whether or not the spot accelerated (or decelerated) as it traversed the screen. Thus, the forced-choice decision was acceleration/no acceleration for the acceleration block, and deceleration/no deceleration for the deceleration block. The observers were informed that the spot could traverse the screen in any of the four directions in any given trial, and they were advised to fixate the center of the screen between trials and maintain their initial distance from the screen. To ensure that the observers understood the task, exemplars of extreme acceleration or deceleration were shown for each motion direction, and the observers were shown how to respond.

The observers were given short breaks between sets, and a longer rest period between blocks. About 2 h were required for an observer to complete the study.

\section{RESULTS}

The observers' threshold values were the dependent measure. Monitor position had no effect on performance and will not be considered further. In addition, threshold values were averaged across the two initial acceleration values (i.e, zero and suprathreshold). The results, averaged across observers, are shown in Table 1 . The magnitude of the threshold values did not differ significantly for acceleration and deceleration $[F(1,7)=3.06$, $p>.10]$, nor was there any reliable effect for average velocity $[F(2,6)=1.39, p>.30$ for the acceleration block; $F(2,6)=2.96, p>.10$ for the deceleration block].

There were, however, significant effects for the direction of motion in both the acceleration and deceleration blocks. Planned comparisons showed that these effects were due to horizontal as opposed to vertical differences $[F(1,7)=8.48, p<.02$ for the acceleration block; $F(1,7)=11.30, p<.02$ for the deceleration block]. The direction of the effect was opposite for motions along the two axes. For horizontal motions, decelerations were easier to detect; for vertical motions, accelerations were more detectable. Thresholds did not differ within principle axes-that is, up versus down $[F(1,7)=5.45, p>.05$ for the acceleration block; $F(1,7)=3.62, p>.10$ for the deceleration block], and left versus right $[F(1,7)=$ $0.56, p>.40$ for the acceleration block; $F(1,7)=0.97$, $p>.30$ for the deceleration block]. The relation between motion axis and type of acceleration is shown in Figure 1.

\section{Table 1}

Mean Threshold Values (Delta $V / A v e r a g e ~ V$ ) as a Function of Acceleration Type, Average Velocity, and Direction of Motion

\begin{tabular}{|c|c|c|c|c|c|c|c|c|}
\hline \multirow{3}{*}{$\begin{array}{l}\text { Average } \\
\text { Velocity* }\end{array}$} & \multicolumn{8}{|c|}{ Direction } \\
\hline & \multicolumn{2}{|c|}{ Up } & \multicolumn{2}{|c|}{ Down } & \multicolumn{2}{|c|}{ Left } & \multicolumn{2}{|c|}{ Right } \\
\hline & $M$ & $S D$ & $M$ & $S D$ & $M$ & $S D$ & $M$ & $S D$ \\
\hline \multicolumn{9}{|c|}{ Acceleration } \\
\hline 0.7 & .72 & .39 & .85 & .45 & .90 & .38 & .80 & .38 \\
\hline 1.2 & .39 & .29 & .55 & .48 & .71 & .37 & .73 & .27 \\
\hline 1.7 & .53 & .20 & .69 & .32 & .83 & .27 & .82 & .36 \\
\hline \multicolumn{9}{|c|}{ Deceleration } \\
\hline 0.7 & -.56 & .33 & -.61 & .36 & -.34 & .28 & -.27 & .24 \\
\hline 1.2 & -.63 & .43 & -.86 & .52 & -.44 & .27 & -.43 & .28 \\
\hline 1.7 & -.62 & .33 & -.82 & .47 & -.54 & .26 & -.45 & .27 \\
\hline
\end{tabular}

*In deg/sec. 


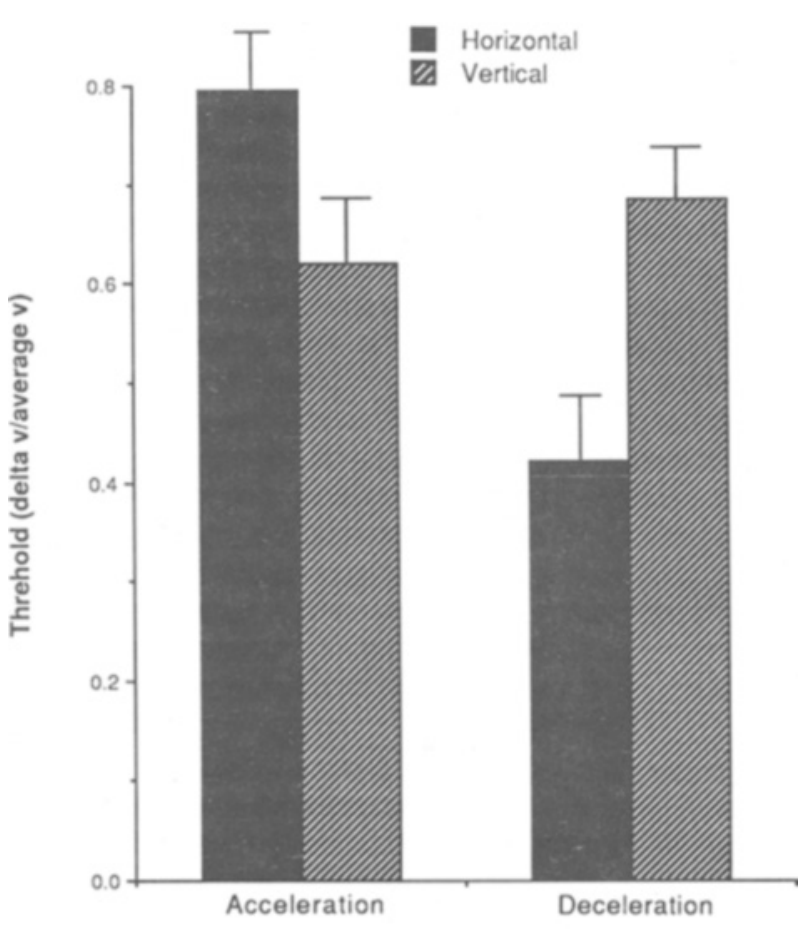

Type of Velocity Change

Figure 1. Mean threshold as a function of motion axis and acceleration sign. Error bars indicate standard error of the estimated means.

\section{DISCUSSION}

The detection thresholds in the present study are slightly smaller than those reported by Schmerler (1976), and they are similar to those reported in Regan et al. (1986) for drifting sinusoidal gratings. (To compare with the values reported by Schmerler, our thresholds were recomputed as final:initial velocity ratios. These ratios ranged from 1.5 to 2.4. The threshold parameter in Regan et al. was the change in velocity divided by the sum of the minimum and maximum velocities, rather than the average. Thus, their values need to be multiplied by 2 for comparison with the present study's findings.) Even if the smallest of these estimates is considered, it is clear that the observers' thresholds for the detection of acceleration are much higher than their limits for the detection of velocity differences.

This discrepancy creates a dilemma for the models of acceleration perception that propose a comparison of early and late velocity segments. Conceivably, such models could be retained if observers either failed to attend to the early part of the event (Gottsdanker, 1961), or if the early and late segments' velocity estimates were influenced by other segments' velocities. This later possibility has been advanced by Schmerler (1976), who suggests that observers have a general tendency to homogenize velocity, just as they homogenize brightness in unbounded fields (Metzger, 1953).

One reason to suspect the inadequacy of an explanation based on initial segment deletion is the present study's finding of a consistent Weber fraction across average velocity. Such a model would predict that, since the higher velocity events occurred in less time, failure to attend to velocity information for a fixed initial interval would more greatly impact these detection thresholds. However, observers were equally sensitive to velocity changes at all levels. Unless one were to propose that the focusing of attention occurs more quickly for high velocity events, it is difficult to defend an initial segment deletion model.

The present data support earlier reports that decelerations are easier to detect than accelerations for motions along the horizontal axis. However, the converse is true for motions along the vertical axis. Such anisotropies are not uncommon in motion perception (see, e.g., Perrone, 1986), but no explanatory mechanism can be given for the phenomenon at this time. This finding that accelerations are, in some conditions, easier to detect than decelerations also suggests that observers do not ignore initial velocity information. Consider that proportionately more change in velocity takes place during the initial segment with accelerations than with decelerations. Therefore, if the initial segment is ignored, decelerations should always be easier to detect. Gottsdanker (1961) has argued for such a model on the basis of his finding that decelerations are easier to detect. However, we found that this holds only for motions along the horizontal axis.

Recent research reported by McKee and Nakayama (1988) supports the velocity homogenization model. They have found that observers are less sensitive to velocity differences if the comparison segment is presented in close temporal proximity (and along the same trajectory axis) as the reference segment. The presence of this "masking" segment can cause a threefold increase in observers' thresholds. Whereas these thresholds are still much smaller than those we report, the observers in McKee and Nakayama's study were highly practiced, and stepfunction changes in velocity were used. Thus, among indirect acceleration detection models, the emerging evidence favors a velocity homogenization (or masking) model over the initial segment deletion model to explain the difficulty that observers experience. Such clarification of the indirect model will make it easier to evaluate such a model against models of direct acceleration detection in future research.

Interestingly, no significant difference was found for detection of accelerations for upward and downward motions. One could imagine that such a bias might exist, given the propensity of objects in the environment to accelerate when moving downward and decelerate when moving upward. Thus, although Shanon (1976) found that people erroneously report that objects in free fall move at a constant velocity, they nonetheless are perceptually sensitive to acceleration in this direction. 
In conclusion, our findings suggest that observers are relatively sensitive to linear accelerations, but less sensitive than comparative velocity judgments would suggest. Thus, the mechanism for acceleration detection does not appear to be a comparison of early and late velocity components, unless velocity homogenization (or masking) occurs. Since there was no significant effect for average velocity, thresholds for acceleration remain a fairly constant ratio of total velocity change over average velocity, at least for the relatively narrow range of velocities examined. However, thresholds did vary as an interactive function of the sign of acceleration and the orientation of the motion axis.

\section{REFERENCES}

Filion, R. D. L. (1964). On the visual detection of accelerated motion. Unpublished doctoral dissertation, Princeton University, Princeton, NJ.

GeIGER, M. (1903). Neue complicationsversuche. Psychologische Studien, 18, 347-436.

GotTSDANKER, R. M. (1955). A further study of prediction-motion. American Journal of Psychology, 68, 432-437.

GOTTSDANKER, R. M. (1961). How the identification of target acceleration is affected by modes of starting and of ending. British Journal . of Psychology, 52, 155-160.

GotTSDANKER, R. M., FrICK, J. W., LockARD, R. B. (1961). Identifying the acceleration of visual targets. British Journal of Psychology, 52, 31-42.

Halloran, T. O., Clark, B., \& Stewart, J. D. (1976). Influence of stimulus size and configuration on pilots' reaction time to accelerating cathode-ray tube displays. Proceedings of the Aerospace Medical Association Annual Scientific Meeting, 186-187.

JAGACINSKI, R. J., Johnson, W. W., Miller, R. A. (1983). Quantifying the cognitive trajectories of extrapolated movements. Journal of Experimental Psychology: Human Perception \& Performance, 9, 43-57.

LeVITT, H. (1970). Transformed up-down methods in psychoacoustics. Joumal of the Acoustical Society of America, 49, 467-477.

McColgin, F. H. (1960). Movement thresholds in peripheral vision. Journal of the Optical Society of America, 50, 774-779.

MCKeE, S. P. (1981). A local mechanism for differential velocity detection. Vision Research, 21, 491-500.
MCKEE, S. P., NAKAYAMA, K. (1988, May). Velocity integration along the trajectory. Paper presented at the Annual Meeting of the Association for Research in Vision and Ophthalmology, Sarasota, FL.

Metzger, W. (1953). Gesetze des Sehens. Frankfurt: WaldemarKramer.

Orban, G. A., Wolf, J. D., \& Maes, H. (1984). Factors influencing velocity coding in the human visual system. Vision Research, 24, 33-39.

Perrone, J. A. (1986). Anisotropic responses to motion toward and away from the eye. Perception \& Psychophysics, 39, 1-8.

Regan, D. M., Kaufman, L., \& Lincoln, J. (1986). Motion in depth and visual acceleration. In K. R. Boff, L. Kaufman, \& J. P. Thomas (Eds.), Handbook of perception and human performance: Vol. 1. Sensory processes and perception (pp. 19-1 to 19-46). New York: Wiley.

Rosenbaum, D. A. (1975). Perception and extrapolation of velocity and acceleration. Journal of Experimental Psychology: Human Perception \& Performance, 1, 395-403.

Runeson, S. (1974). Constant velocity-not perceived as such. Psychological Research, 37, 3-23.

Shanon, B. (1976). Aristotelianism, Newtonianism, and the physics of the layman. Perception, 5, 241-243.

SChmeRLeR, J. (1976). The visual perception of accelerated motion. Perception, 5, 167-185.

WUNDT, W. (1874). Grundzuge der physiologischen Psychologie. Leipzig: Engelmann.

\section{NOTES}

1. Different initial step sizes were used for the three average velocity conditions: $0.12 \% \mathrm{sec}^{2}, 0.38 \% / \mathrm{sec}^{2}$, and $0.77^{\circ} / \mathrm{sec}^{2}$. Step size was decreased on alternate reversals to $I / N$, where $I$ is the initial step size and $N$ is the number of reversals. Staircases were terminated at the ninth reversal, and midrun averages were used for threshold estimates. An excellent discussion of the efficiency of staircase methodologies for threshold localization is provided in Levitt (1970) and the references cited therein.

2. Like most microcomputer monitors, the Amiga's does not have a 1:1 aspect ratio. The graphics program compensated for this resolution difference such that the motion parameters were equivalent for the horizontal and vertical axes. Nonetheless, monitor orientation was varied across subjects to ensure that screen artifacts did not bias performance.

(Manuscript received December 4, 1987; revision accepted for publication October 12, 1988.) 